\title{
Handlungsstandards fürr die Assistenz in der Endoskopie
}

In dieser Ausgabe beginnen wir eine Serie zur neuen Techniken. Wir starten mit der Therapie von Nahtinsuffizienten mittels Schwammtherapie, gefolgt von Techniken zum Lackagenverschluss.

\section{Hinweis}

Bei den hier abgedruckten Standards handelt sich nicht um nationale Standards oder Expertenstandards. Die hier vorgestellten Arbeitshilfen oder Mikrostandards sind so allgemein formuliert, damit sie jeder Abteilung adaptiert werden können. Die Standards beinhalten nur die wichtigsten Informationen.

\section{Nutzung dieses Standards}

Das DIN-A6-Format passt in die Kitteltaschen der üblichen Dienst- und Bereichskleidung. Dadurch können diese Standards von jedem Leser in der täglichen Routine genutzt werden, z.B. wenn

- neue Kolleginnen und Kollegen angelernt werden,

- neue Techniken in der Abteilung eingeführt werden,

- Techniken selten durchgeführt werden und eine Gedankenstütze in der Vorbereitung und Durchführung hilfreich ist.
Folgende Vorgehensweise ist für die Nutzung der Standards vorgesehen:

- Schneiden Sie den Standard an der gestrichelten Linie aus.

- Danach falten Sie den Standard in der Mitte, sodass Sie Vorderund Rückseite des Standards lesen können.

- Laminieren Sie den Standard in dem nun entstandenen DINA6-Format.

Ihre Wünsche sind uns wichtig

Wir möchten Ihre Wünsche bei zukünftigen Standards gerne berücksichtigen. Wir laden Sie daher sehr herzlich ein, Themen für einzelne Standards oder Themenkomplexe vorzuschlagen. Bitte senden Sie Ihre Kommentare und Vorschläge an Ulrike Beilenhoff, E-Mail: UK-Beilenhoff@t-online.de

Wir freuen uns auf Ihre Rückmeldungen

Ulrike Beilenhoff E Ute Pfeifer

\section{EsO-SPONGE ${ }^{\circledR}$}

\section{Einsatzgebiet}

Endoluminale Vakuumtherapie zur Behandlung der Anastomoseninsuffizienz des oberen GI-Trakts

\section{Indikationen}

- nach chirurgischen Eingriffen mit Anastomosen

- nach endoskopischen Interventionen

durch Boerhaave-Syndrom

Varianten

- Intraluminale Schwammdrainage

- intrakavitäre Schwammdrainage

- Kombination beider Techniken

Allgemeine Vorbereitung

- identisch zur diagnostischen ÖGD

- größeren Zeitkorridor einplanen

Vorbereitung des Patienten

> in Links-Seitenlage

- in Propofol- oder Analgosedierung mit Intubation/Beatmung

Untersuchungsvorbereitung

- Gastroskop mit Außendurchmesser 11,2 mm

- Magensonde Ch.16,Hydrogel, $20 \mathrm{ml}$ steriles $\mathrm{NaCl}$ 0,9\%

- Pean-Klemmen/Schere/transparentes Tegaderm ${ }^{\mathrm{TM}}$

- Vakuumpumpe mit Auffangbehälter

- Eso-SPONGE ${ }^{\circledR}$-Sets in verschiedenen Größen $13-17 \mathrm{~mm} /$ $15-19 \mathrm{~mm}$

> optional: verschiedenstufige Dilatationsballons, mit Druckspritze und Manometer für evtl. Dilatation

Merke: $\mathrm{CO}_{2}$ verwenden

Vorbereitung des sterilen Instrumenten-Tisches

- Inhalt des Sets anordnen: Eso-SPONGE ${ }^{\circledR}$, Overtube, Pusher, Spül-Set und $20 \mathrm{ml} \mathrm{NaCl} \mathrm{0,9 \% ,} \mathrm{Schere} \mathrm{und} \mathrm{Klemmen,} \mathrm{Y-Verbin-}$ dungsstück

\section{Durchführung}

- nach endoskopischer Untersuchung und Ausmessen der Leckage Platzierung des Eso-SPONGE ${ }^{\circledR}$ mittels Endoskop-Overtube und Pusher in die Wundhöhle, Freisetzen des Schwamms, endoskopische Lagekontrolle und ggf. Korrektur mit Fasszange

- Transnasale Ausleitung: Magensonde CH.16 durch Nase einführen und Ausleiten durch Mund, Abschneiden der atraumatischen Spitze

- Konnektierung des Drains mit Magensonde vor dem Mund

- Y-Stück und Schwammdrain mit Tegaderm ${ }^{\mathrm{TM}}$ umkleben und an Vakuumpumpe anschließen, Sog: kontinuierlich nach Arztangaben

Endoskopische Wundkontrollen und Schwammwechsel

- alle 48-72 Stunden, hierzu wird der Sog unterbrochen

- zum Entfernen des Schwamms das Drain wieder umleiten

- Anspülen des Schwammdrains mit $20 \mathrm{ml} \mathrm{NaCl} \mathrm{0,9 \%} \mathrm{und}$ Spülset, Schwamm löst sich aus der Wundhöhle

\section{Ernährung}

- intraluminale Anlage $\Rightarrow$ Ernährungssonde, PEG

> intrakavitäre Anlage $\Rightarrow$ oral, am Drain vorbei

Komplikationen

- schwerwiegende Komplikationen sind selten

mögliche Risiken sind Blutungen durch Gefäßarrosionen sowie Entstehung einer Fistel

Kleinere Probleme sind eher technischer Natur:

- Schwammdislokation

- partielle Verstopfung des Schwamms und dadurch insuffiziente Sogwirkung

- Abriss des Schwamms beim Systemwechsel, Extraktion mittels Fasszange

Petra Holzky-Haselbach, UKSH Campus Kiel 